\title{
Effect of Edaravone Combined with Anticoagulant Therapy on the Serum hs-CRP, IL-6, and TNF- $\alpha$ Levels and Activity of Daily Living in Patients with Acute Cerebral Infarction
}

\author{
Chao Sun, ${ }^{1}$ Cuiling $\mathrm{Ma}^{2}{ }^{2}$ Yu Sun, ${ }^{1}$ and Lili $\mathrm{Ma} \mathbb{C}^{1}$ \\ ${ }^{1}$ Department of Neurology, Yantaishan Hospital, Yantai 264000, Shandong, China \\ ${ }^{2}$ Department of Neurology, The People's Hospital of Feicheng, Feicheng 271600, Shandong, China \\ Correspondence should be addressed to Lili Ma; malili@ytsyy.org.cn
}

Received 31 December 2021; Revised 13 January 2022; Accepted 7 February 2022; Published 28 February 2022

Academic Editor: Bhagyaveni M.A

Copyright (c) 2022 Chao Sun et al. This is an open access article distributed under the Creative Commons Attribution License, which permits unrestricted use, distribution, and reproduction in any medium, provided the original work is properly cited.

Objective. To explore the effect of edaravone combined with anticoagulant therapy on the serum hs-CRP, IL-6, and TNF- $\alpha$ levels and the activity of daily living (ADL) in patients with acute cerebral infarction (ACI). Methods. The clinical data of 84 ACI patients treated in our hospital from August 2020 to August 2021 were retrospectively analyzed, and they were divided into the routine group $(n=42)$ and the combined group $(n=42)$ according to the order of admission. Both groups were treated with routine clinical treatment, and the combined group was additionally treated with edaravone combined with anticoagulant therapy. Serum samples were collected from both groups after treatment. ELISA was used to detect the serum inflammatory factor levels, and the modified Barthel index score was used to evaluate the ADL of patients. Results. Compared with the routine group, the combined group achieved obviously lower levels of PMA, CD62p, and serum inflammatory factors after treatment $(P<0.001)$, higher modified Barthel score after treatment $(P<0.001)$, lower plasma viscosity, platelet aggregation rate, and plasma fibrinogen level after treatment $(P<0.001)$, and higher clinical overall efficacy $(P<0.05)$. Conclusion. Edaravone combined with anticoagulant therapy is a reliable method to enhance ADL and reduce the inflammatory response of ACI patients. This strategy greatly reduces the platelet-activating factor levels of patients and improves the comprehensive clinical efficacy, and its further research will help to establish a better solution for these patients.

\section{Introduction}

Acute cerebral infarction (ACI) refers to brain tissue necrosis caused by the sudden interruption of cerebral blood supply, with complicated pathogenesis [1]. Its etiology may be related to cerebral artery stenosis and blockage caused by abnormal blood vessels, blood, and hemodynamics [2]. With acute onset, rapid progression, and the clinical manifestations of most patients as tinnitus, headache, slurred speech, and vomiting, this disease damages the neurological function and leads to a poor prognosis. With the aggravation of aging and lifestyle changes in China, the clinical incidence of $\mathrm{ACI}$ has been rising by year and developing toward a younger age, and its clinical diagnosis and treatment have become the focus of social concern [3]. A large number of clinical studies $[4,5]$ have confirmed that effective dredging of infarction lesions and improvement of neurological function at an early stage are the key factors affecting the prognosis of patients. At present, antiplatelet drugs such as aspirin, ozagrel, and clopidogrel are mostly used in clinic, but the efficacy remains unsatisfactory [6]. Edaravone, a free radical scavenger, can improve the local blood flow of cerebral infarction lesions, prevent the disease progression, and alleviate neurological symptoms, with confirmed efficacy in diseases such as acute carbon monoxide poisoning, cardiogenic cerebral infarction, and hypertensive cerebral hemorrhage [7-9]. Although not recommended in the guidelines, anticoagulant therapy for ACI is very common in foreign countries, with the common anticoagulant drugs such as aspirin, warfarin, and plavix. From the perspective of pathophysiological mechanism, anticoagulant therapy can restrain the deterioration of neurological symptoms, prevent 
the recurrence of early stroke, and improve microcirculation and blood supply [10]. However, the current research on anticoagulant therapy for ACI is still in the initial stage, lacking sufficient evidence-based proof [11]. At present, few studies have reported the efficacy of edaravone combined with anticoagulant therapy on ACI. Based on this, the ACI patients admitted to our hospital were retrospectively analyzed and received different treatment regimens after admission, aiming to observe whether edaravone combined with anticoagulant therapy can benefit ACI patients in terms of clinical effect, reported as follows.

\section{Materials and Methods}

2.1. Clinical Data. The clinical data of 84 ACI patients treated in our hospital from August 2020 to August 2021 were retrospectively analyzed, and they were divided into the routine group $(n=42)$ and the combined group $(n=42)$ according to the order of admission. This study was in line with the Declaration of Helsinki (as revised in 2013) [12].

Inclusion criteria are as follows: (1) the enrolled subjects all met the diagnostic criteria of ACI in Chinese Guidelines for Diagnosis and Treatment of Acute Ischemic Stroke (2010) [13], and the infarction lesions were confirmed by MRI or CT scan, with the CT images showing low-density lesions and the symptoms such as tinnitus, vertigo, nausea, vomiting, and coma; (2) all patients were treated within 72 hours after onset, and had the first onset; and (3) patients had no allergic history for the drugs used in this treatment, with clear consciousness.

Exclusion criteria are as follows: (1) the patients with serious injury of other vital organs; (2) patients complicated with coagulation disorders; (3) patients who had received anticoagulant treatment before; and (4) patients who had the diseases with hemorrhagic tendency.

2.2. Treatment Methods. Both groups received routine treatment after hospitalization, including basic treatments such as lowering intracranial pressure, regulating waterelectrolyte imbalance, improving microcirculation, antihypertension, and oxygen inhalation.

The combined group was additionally treated with edaravone combined with anticoagulant therapy. $30 \mathrm{mg}$ of edaravone (manufacturer: China National Medicines Guorui Pharmaceutical Co., Ltd.; NMPA approval no.: H20080056; specification: $20 \mathrm{ml} / 30 \mathrm{mg}$ ) was dissolved in $100 \mathrm{ml}$ of isotonic saline and then was intravenously dripped into the patients for continuous treatment of 14 days, twice a day. At the same time, anticoagulant therapy was performed through the subcutaneous injection of $5000 \mathrm{IU}$ of low molecular weight heparin calcium (manufacturer: Hebei Changshan Biochemical Pharmaceutical Co., Ltd.; NMPA approval no.: H20063909; specification: $0.2 \mathrm{ml}$ : 2050AXaIUx2 vials/box) for continuous treatment of 14 days, once every 12 hours.
2.3. Observation Indexes. Fasting elbow venous blood $(5 \mathrm{~mL})$ was collected in the morning from both groups after treatment and centrifuged at $3000 \mathrm{r} / \mathrm{min}$ for $15 \mathrm{~min}$. The serum was taken and stored in a refrigerator at $-80^{\circ} \mathrm{C}$ for detection. The XTG-1600E flow cytometry (manufacturer: Shanghai Huanxi Medical Device Co., Ltd.) was used to detect the serum platelet-activating factors, including the levels of platelet-monocyte aggregation (PMA) and $\alpha$-granulosa glycoprotein (CD62p). ELISA was adopted to detect the serum inflammatory factor levels, including interleukin-6 (IL-6), high-sensitivity C-reactive protein (hsCRP), and tumor necrosis factor- $\alpha$ (TNF- $\alpha)$. The kits were purchased from Shenzhen Xinbosheng Biological Co., Ltd., and the detection was performed strictly in accordance with the kit instructions.

Hemorheology indexes: $4 \mathrm{ml}$ of peripheral venous blood was collected after treatment, and a hemorheological analyzer (manufacturer: Dongguan Maiyue Medical Device Co., Ltd.; model: HL-5000) was used to detect the plasma fibrinogen, platelet aggregation rate, and plasma viscosity.

The modified Barthel Index Scale [14] was used to evaluate the activity of daily living (ADL) of patients, including 11 items such as diet, bathing, dressing, and walking. Each activity was rated in five levels, and different levels represented different levels of independent ability. The lowest level was level 1, and the highest level was level 5 , and a higher level indicated higher independent ability. The patients with a score $\geq 60$ points could basically take care of themselves; those with 41-59 had moderate dysfunction and needed help from others in daily life; those with 21-40 had severe dysfunction, with obviously life dependence; and those with a score $\leq 20$ were completely dependent on others. The specific scoring criteria are shown in Table 1.

Evaluation of overall efficacy after treatment: the European Stroke Scale (ESS) [15] was used to evaluate the clinical efficacy of patients before and after treatment. The scale had 14 items, including consciousness level, language, visual field, finger muscle strength, and gait, with a total score of 100 points. A higher score represented a better function of the subjects.

If the ESS score increased by $85 \%-100 \%$, the patients were cured; if the ESS score increased by $50 \%-84 \%$, the treatment was markedly effective; if the ESS score increased by $20 \%-49 \%$, the treatment was effective; and if the ESS score increased by less than $20 \%$, the treatment was ineffective. Total effective rate $=($ cured cases + markedly effective cases)/total cases $\times 100 \%$.

2.4. Statistical Methods. The data in the study were processed by the professional statistical software SPSS23.0 and graphed by GraphPad Prism 7 (GraphPad Software, San Diego, USA). The enumeration data were tested by $\chi^{2}$ and expressed as $n(\%)$, while the measurement data were tested by the $t$ test and expressed as mean \pm SD. When $P<0.05$, the differences were statistically significant. 
TABLe 1: Scoring criteria of modified Barthel Index Scale.

\begin{tabular}{|c|c|c|c|c|c|}
\hline Assessment items & $\begin{array}{c}\text { Complete dependence } \\
\text { level } 1\end{array}$ & $\begin{array}{l}\text { Maximum help } \\
\text { level } 2\end{array}$ & $\begin{array}{l}\text { Medium help } \\
\text { level } 3\end{array}$ & $\begin{array}{c}\text { Minimum help } \\
\text { level } 4\end{array}$ & $\begin{array}{c}\text { Complete independence } \\
\text { level } 5 \\
\end{array}$ \\
\hline Diet & 0 & 2 & 5 & 8 & 10 \\
\hline Defecation & 0 & 2 & 5 & 8 & 10 \\
\hline Bathing & 0 & 1 & 3 & 4 & 5 \\
\hline Decoration & 0 & 1 & 3 & 4 & 5 \\
\hline Walking & 0 & 3 & 8 & 12 & 15 \\
\hline Stool control & 0 & 2 & 5 & 8 & 10 \\
\hline Urine control & 0 & 2 & 5 & 8 & 10 \\
\hline Stairs climbing & 0 & 2 & 5 & 8 & 10 \\
\hline $\begin{array}{l}\text { Movement of beds and } \\
\text { chairs }\end{array}$ & 0 & 3 & 8 & 12 & 15 \\
\hline Dressing & 0 & 2 & 5 & 8 & 10 \\
\hline Sitting in a wheelchair* & 0 & 1 & 3 & 4 & 5 \\
\hline
\end{tabular}

*indicated that this item was assessed only when the patients were not able to walk.

\section{Results}

3.1. Comparison of Clinical Data. No notable differences in clinical data such as gender ratio, onset time, diseased sites, and disease types were observed between the two groups $(P>0.05)$, see Table 2 .

3.2. Comparison of Platelet-Activating Factors. After treatment, the PMA and CD62p levels in the combined group were obviously lower compared with the routine group $(P<0.001)$, see Figure 1.

3.3. Comparison of Serum Inflammatory Factor Levels. After treatment, the various serum inflammatory factor levels were obviously lower in the combined group than in the routine group $(P<0.001)$, see Table 3 .

3.4. Comparison of Hemorheology Indexes. After treatment, plasma viscosity, platelet aggregation rate, and plasma fibrinogen level in the combined group were remarkably lower compared with the routine group $(P<0.001)$, see Table 4.

3.5. Comparison of Modified Barthel Scores. The modified Barthel score in the combined group after treatment was obviously higher compared with the routine group $(P<0.001)$, see Figure 2 .

3.6. Comparison of Overall Efficacy after Treatment. The total clinical effective rate of the combined group was markedly higher than the routine group $(P<0.05)$, see Table 5 .

\section{Discussion}

Relevant epidemiological investigation [16] shows that ACI has become the second leading cause of death worldwide. As a common and frequently-occurring disease in clinic, ACI is characterized by high morbidity, mortality, disability, and recurrence. The abnormal blood supply of local brain tissues caused by various factors leads to brain tissue lesions and damages the brain tissues, triggering ACI. With acute onset and rapid progression, ACI patients have a poor prognosis and are prone to sequelae such as hemiplegia, hemiparesis, and language barriers, seriously affecting their physical and mental health and quality of life [17]. At present, ACI is mainly treated with anticoagulation, thrombolysis, and lipid-lowering to stabilize plaques. The common side effect of thrombolytic therapy is bleeding, which can manifest as skin and mucosa bleeding in patients with mild symptoms and visceral hemorrhage, such as gastrointestinal bleeding and brain bleeding, in patients with severe symptoms. Lipidlowering drugs to stabilize plaque drugs often lead to gastrointestinal adverse reactions, such as upper abdominal discomfort and nausea as well as muscle weakness or pain, in a small number of patients. A study [18] has found that fibrinogen increases and the body is in a hypercoagulable state in ACI patients because the impaired vascular endothelium triggers large consumption of anticoagulant factors such as antithrombin III (AT3) in the fibrinolytic process, promoting the formation of the hypercoagulable state to a certain extent. Due to the hypercoagulable state, low anticoagulant state, and fibrinolytic dysfunction, the reduced blood perfusion in the ischemic area, poor collateral circulation compensation, and expansion of thrombosis will result in the disease progression [19], suggesting that anticoagulant therapy should be given in time after the onset of ACI. However, conventional anticoagulant therapy may increase the risk of bleeding complications. Therefore, it is still controversial about anticoagulant therapy for acute ACI.

A clinical study [20] has shown that the short-term application of low molecular weight heparin in mild ACI is safe and effective. The effect of the anticoagulant factor Xa of low molecular weight heparin is $2-4$ times that of unfractionated heparin, while the anticoagulant factor IIa has a weak effect, so the incidence of bleeding risk is significantly lower than that of unfractionated heparin. A domestic clinical control trial [21] showed that after patients in the control group orally took $100 \mathrm{mg}$ of aspirin once a day, while the observation group additionally received the subcutaneous injection of low molecular weight heparin (5000 IU), twice a day, the National Institutes of Health Stroke Scale (NIHSS) score and cerebral infarction improvement in the 
TABLE 2: Comparison of clinical data $(n=42)$.

\begin{tabular}{|c|c|c|c|c|}
\hline Items & Routine group & Combined group & $\chi^{2} / t$ & $P$ value \\
\hline Gender & & & 0.192 & 0.661 \\
\hline Male/female & $24 / 18$ & $22 / 20$ & & \\
\hline Body weight (mean $\pm \mathrm{SD}, \mathrm{kg}$ ) & $73.16 \pm 5.43$ & $73.41 \pm 5.90$ & 0.202 & 0.840 \\
\hline Average age (mean $\pm S D$, years old $)$ & $60.38 \pm 3.77$ & $60.74 \pm 3.39$ & 0.460 & 0.647 \\
\hline Onset time (mean $\pm S D, h)$ & $23.45 \pm 4.61$ & $23.83 \pm 3.94$ & 0.406 & 0.686 \\
\hline \multicolumn{5}{|l|}{ Diseased sites } \\
\hline Brainstem & $15(35.71)$ & $13(30.95)$ & 0.214 & 0.643 \\
\hline Internal capsule & $21(50.00)$ & $19(45.24)$ & 0.191 & 0.662 \\
\hline Lateral frontotemporal lobe & $6(14.29)$ & $10(23.81)$ & 1.235 & 0.266 \\
\hline \multicolumn{5}{|l|}{ Combined diseases } \\
\hline Hyperlipidemia & $8(19.05)$ & $9(21.43)$ & 0.074 & 0.786 \\
\hline Hypertension & $14(33.33)$ & $12(28.57)$ & 0.223 & 0.637 \\
\hline Coronary heart disease & $15(35.71)$ & $13(30.95)$ & 0.214 & 0.643 \\
\hline Diabetes & $5(11.90)$ & $8(19.05)$ & 0.819 & 0.365 \\
\hline Smoking history & $13(30.95)$ & $16(38.10)$ & 0.474 & 0.491 \\
\hline Drinking history & $17(40.48)$ & $19(45.24)$ & 0.194 & 0.659 \\
\hline \multicolumn{5}{|l|}{ Disease types } \\
\hline Monofocal type & $18(42.86)$ & $16(38.10)$ & 0.198 & 0.657 \\
\hline Multifocal type & $10(23.81)$ & $13(30.95)$ & 0.539 & 0.463 \\
\hline Lacunar type & $14(33.33)$ & $13(30.95)$ & 0.055 & 0.815 \\
\hline \multicolumn{5}{|l|}{ Marital status } \\
\hline Married & $35(83.33)$ & $37(88.10)$ & 0.389 & 0.533 \\
\hline Unmarried & $4(9.52)$ & $3(7.14)$ & 0.156 & 0.693 \\
\hline Divorced & $3(7.14)$ & $2(4.76)$ & 0.213 & 0.645 \\
\hline Residence & & & 0.192 & 0.661 \\
\hline Urban area & $18(42.86)$ & $20(47.62)$ & & \\
\hline Rural area & $24(57.14)$ & $22(52.38)$ & & \\
\hline
\end{tabular}

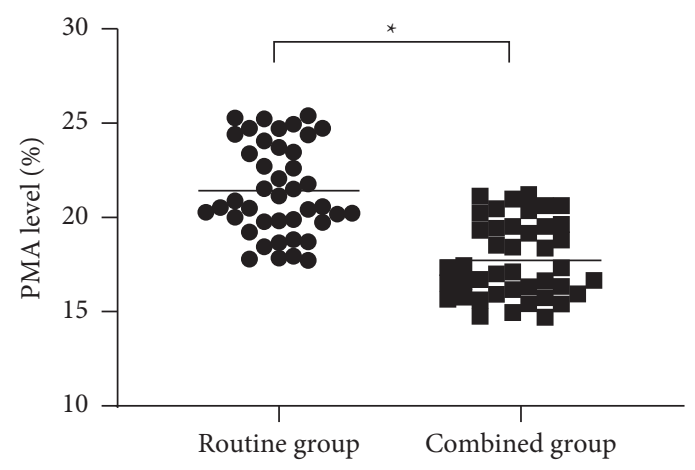

(a)

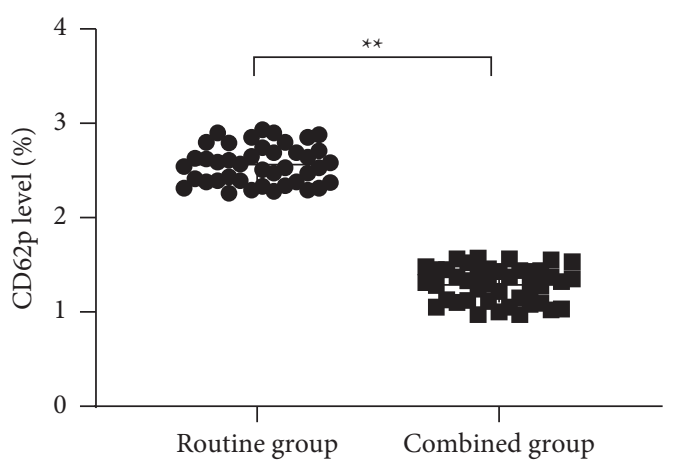

(b)

Figure 1: Comparison of platelet-activating factors after treatment (mean \pm SD). (a) comparison of PMA levels after treatment. The abscissa represented the routine group and combined group, and the ordinate represented the PMA level (\%). The average PMA levels of the routine group and combined group after treatment were $(21.42 \pm 2.42) \%$ and $(17.72 \pm 2.00) \%$. ${ }^{*}$ indicated a notable difference in the average PMA levels between the two groups after treatment $(t=7.638, P<0.001)$. (b) Comparison of CD62p levels after treatment. The abscissa represented the routine group and combined group, and the ordinate represented the CD62p level (\%). The average CD62p levels of the routine group and combined group after treatment were $(2.56 \pm 0.20) \%$ and $(1.29 \pm 0.19) \%{ }^{* *}$ indicated a notable difference in the average CD62p levels between the two groups after treatment $(t=29.836, P<0.001)$.

TABLE 3: Comparison of serum inflammatory factor levels after treatment (mean $\pm S D$ ).

\begin{tabular}{lcccc}
\hline Group & $n$ & hs-CRP $(\mu \mathrm{g} / \mathrm{L})$ & IL-6 $(\mathrm{ng} / \mathrm{L})$ & TNF- $\alpha(\mathrm{ng} / \mathrm{L})$ \\
\hline Routine group & 42 & $16.52 \pm 1.38$ & $62.90 \pm 3.04$ & $287.87 \pm 13.31$ \\
Combined group & 42 & $10.77 \pm 1.41$ & $48.15 \pm 2.95$ & $132.95 \pm 12.78$ \\
$t$ & & 18.888 & 22.566 & 54.411 \\
$P$ value & & $<0.001$ & $<0.001$ & $<0.001$ \\
\hline
\end{tabular}


TABLE 4: Comparison of hemorheology indexes after treatment (mean $\pm \mathrm{SD})$.

\begin{tabular}{lcccc}
\hline Group & $n$ & Plasma viscosity $(\mathrm{MPa} \cdot \mathrm{s})$ & Platelet aggregation rate $(\%)$ & Plasma fibrinogen $(\mathrm{g} / \mathrm{L})$ \\
\hline Routine group & 42 & $5.54 \pm 0.59$ & $55.44 \pm 5.96$ & $5.34 \pm 0.68$ \\
Combined group & 42 & $4.36 \pm 0.65$ & $48.17 \pm 6.06$ & $3.46 \pm 0.67$ \\
$t$ & & 8.711 & 5.543 & 12.763 \\
$P$ value & $<0.001$ & $<0.001$ & $<0.001$ \\
\hline
\end{tabular}

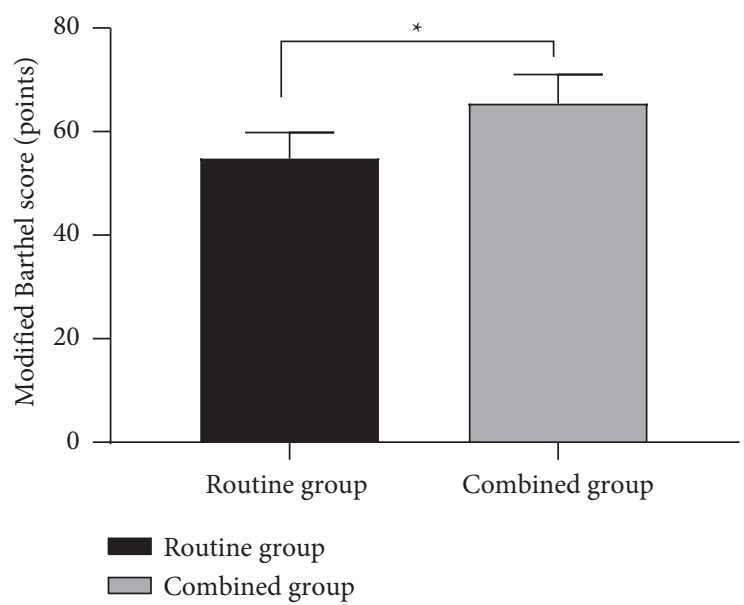

FIGURE 2: Comparison of modified Barthel scores after treatment (mean \pm SD). The abscissa represented the routine group and combined group, and the ordinate represented the modified Barthel score (points). The modified Barthel scores of the routine group and combined group after treatment were $(54.86 \pm 4.96)$ and $(65.40 \pm 5.61) .{ }^{*}$ indicated a notable difference in the modified Barthel scores after treatment between the two groups $(t=9.122, P<0.001)$.

TABLE 5: Comparison of overall efficacy after treatment $(n(\%))$.

\begin{tabular}{|c|c|c|c|c|c|c|}
\hline Group & $n$ & Cured & Markedly effective & Effective & Ineffective & Total effective rate \\
\hline Combined group & 42 & $13(30.95)$ & $24(57.14)$ & $3(7.14)$ & $2(4.76)$ & $88.10 \%(37 / 42)$ \\
\hline Routine group & 42 & $11(26.19)$ & $18(42.86)$ & $8(19.05)$ & $5(11.90)$ & $69.05 \%(29 / 42)$ \\
\hline & & & & & & 4.525 \\
\hline$P$ value & & & & & & $<0.05$ \\
\hline
\end{tabular}

observation group after treatment were better than those in the control group $(P<0.05)$, which confirmed the better effect of anticoagulant therapy. While removing the free radicals in the injured parts, edaravone, a neuroprotective agent, can reduce the damage and apoptosis of nerve cells, improve the survival of nerve cells in the ischemic parts of the brain and reduce the damaged area of cerebral infarction, thus promoting the recovery of blood supply for injured brain tissues [22].

Hypoxia, ischemia, and blood supply disorders in local brain tissues can activate microglia and peripheral white blood cells, resulting in a series of inflammatory reactions in the body and aggravating the damage of brain tissues and peripheral neurons. A previous study [23] has shown that inflammatory factors such as IL-6, IL-8, and hs-CRP can promote the formation of thrombosis, among which hs-CRP is an important indicator reflecting atherosclerosis and acute inflammatory response. In this study, after edaravone combined with anticoagulant therapy was adopted, the serum inflammatory factor levels in the combined group after treatment were notably lower than those in the routine group $(P<0.001)$, suggesting that the combined therapy can effectively alleviate the inflammatory response of ACI patients and then delay the disease progression. ACI patients have a high risk of sequelae for the special nerve cells, resulting in the decrease of ADL in patients. Therefore, effective treatment programs to improve the ADL of patients have become the key to improving the prognosis of the disease [24]. In this study, it was found that the modified Barthel score after treatment was obviously higher in ACI patients who received edaravone combined with anticoagulant therapy than in those with routine treatment, indicating that the combined therapy can reduce perfusion injury of cerebral cells and improve the ADL of patients. The study also has some shortcomings. For example, the small sample size of ACI patients indicates the possible selection bias. In addition, the adverse reactions of patients after treatment are not studied. Therefore, it is necessary to expand the sample size and conduct more multicenter studies to further confirm the results of the study.

In conclusion, edaravone combined with anticoagulant therapy is a reliable method to reduce the serum 
inflammatory factor levels, improve the hemorheology indexes, and enhance the ADL of ACI patients. Therefore, its further research will help to establish a better solution for such patients.

\section{Data Availability}

The data used to support the findings of this study are available on reasonable request from the corresponding author.

\section{Conflicts of Interest}

The authors have no conflicts of interest to declare.

\section{References}

[1] L. Shi, K. Wang, X. Duan et al., "Efficacy of danshen class injection in the treatment of acute cerebral infarction: a bayesian network meta-analysis of randomized controlled trials," Evid Based Complement Alternat Med, vol. 2019, Article ID 5814749, 11 pages, 2019.

[2] Z. Li, X. Rong, J. Luo, T. Zeng, P. Huang, and X. Xu, "A singlecenter clinical study to evaluate shenxiong glucose injection combined with edaravone in the treatment of acute large-area cerebral infarction," BioMed Research International, vol. 2021, Article ID 9935752, 2021.

[3] Z. Sun, Q. Xu, G. Gao, M. Zhao, and C. Sun, "Clinical observation in edaravone treatment for acute cerebral infarction," Nigerian Journal of Clinical Practice, vol. 22, no. 10, pp. 1324-1327, 2019.

[4] H. Bao, H.-R. Gao, M.-L. Pan, L. Zhao, and H.-B. Sun, "Comparative study on the efficacy and safety of alteplase and urokinase in the treatment of acute cerebral infarction," Technology and Health Care, vol. 29, no. 1, pp. 85-90, 2021.

[5] F. Sun, H. Liu, H. X. Fu et al., "Comparative study of intravenous thrombolysis with rt-PA and urokinase for patients with acute cerebral infarction," Journal of International Medical Research, vol. 48, Article ID 300060519895352, 2020.

[6] Y. Liu, J. Yang, P. Jiang et al., "DAPT score: predictive model of dual-antiplatelet therapy for acute cerebral infarction," Neurological Sciences, vol. 42, no. 2, pp. 681-688, 2021.

[7] J. Guo, J. Wang, Y. Guo, and J. Feng, "Association of aspirin resistance with 4-hydroxynonenal and its impact on recurrent cerebral infarction in patients with acute cerebral infarction," Brain and behavior, vol. 10, Article ID e01562, 2020.

[8] L. Xiu-Xiu, L. Shi-Hui, Z. Su-Jing, S. Guo, and S. Pang, "Effects of intravenous thrombolysis with alteplase combined with edaravone on cerebral hemodynamics and T lymphocyte level in patients with acute cerebral infarction," Journal of Medicine, vol. 99, Article ID e23414, 2020.

[9] T. Wu, P. Li, and D. Sun, "Assessing the clinical efficacy of recombinant tissue plasminogen activator on acute cerebral infarction," Journal of Nanoscience and Nanotechnology, vol. 20, no. 12, pp. 7781-7786, 2020.

[10] J. Zeng, F. Wang, F. Chen et al., "Influencing factors of recanalization after intravenous thrombolysis with urokinase in acute cerebral infarction patients," European Neurology, vol. 83, no. 2, pp. 162-166, 2020.

[11] Y. Y. Dang, Z. H. Zhang, and C. S. Shen, "Carotid artery stenting for the treatment of fatal acute cerebral infarction associated with aortic dissection," Neurology India, vol. 68, pp. 185-188, 2020.
[12] World Medical Association, "World medical association declaration of Helsinki: ethical principles for medical research involving human subjects," JAMA, vol. 310, no. 20, pp. 2191-2194, 2013.

[13] O. Takeshi, K. Koh, K. Masateru, and J. Duncan, "Perioperative management of patients with atrial fibrillation receiving anticoagulant therapy," Journal of Anesthesia, vol. 33, pp. 551-561, 2019.

[14] H. Yamaji, S. Higashiya, T. Murakami et al., "Effects of oral anticoagulants on patients with atrial fibrillation aged 90 Years and older," Journal of Cardiovascular Pharmacology, vol. 74, no. 3, pp. 246-254, 2019.

[15] Q. Chen, J. Ke, X. Cai et al., "GABA-induced motor improvement following acute cerebral infarction," American Journal of Tourism Research, vol. 12, no. 12, pp. 7724-7736, 2020.

[16] X. L. Zhang, Y. T. Dong, Y. Liu, Y. Zhang, T. T Li, and F. Y. Hu, "Effects of dl-3-n-butylphthalide on serum lipoprotein-associated phospholipase A2 and hypersensitive C-reactive protein levels in acute cerebral infarction," Brain and behavior, vol. 9, Article ID e01469, 2019.

[17] M. Li, J. Wang, X. Wang, and G. Li, "Clinical efficacy of aspirin combined with clopidogrel in treating cerebral infarction and its effect on serum hs-CRP, sICAM-1 and TNF- $\alpha$," Experimental and Therapeutic Medicine, vol. 19, pp. 939-944, 2020.

[18] Y. Chen and Y. Zhao, "Curative efficacy of penehyclidine combined with edaravone on acute cerebral infarction and their effects on serum TNF- $\alpha$ and NDS score in rats," European Review for Medical and Pharmacological Sciences, vol. 22, pp. 223-228, 2018.

[19] L. Chen, Q. Yang, R. Ding, D Liu, and Z Chen, "Carotid thickness and atherosclerotic plaque stability, serum inflammation, serum MMP-2 and MMP-9 were associated with acute cerebral infarction," Experimental and Therapeutic Medicine, vol. 16, pp. 5253-5257, 2018.

[20] S. Guo, L. Ai-Hong, C. Xin, and L. Sun, "Effect of acupoint catgut embedding on motor function and serum high sensitivity C-reactive protein and IL-6 levels in patients with acute cerebral infarction," Zhen Ci Yan Jiu, vol. 38, pp. 224-228, 2013.

[21] C. Yu, W. Wang, Y. Zhang et al., "The effects of modified constraint-induced movement therapy in acute subcortical cerebral infarction," Frontiers in Human Neuroscience, vol. 11, p. 265, 2017.

[22] K. Amira Adly, A. Adel Mohamed, and S. A. Mohamed, "Edaravone attenuates lung injury in a hind limb ischemiareperfusion rat model: a histological, immunohistochemical and biochemical study," Ann Anat, vol. 228, p. 151433, 2020.

[23] X. Zhang, G. Ye, Z. Wu et al., "The therapeutic effects of edaravone on collagen-induced arthritis in rats," Journal of Cellular Biochemistry, vol. 121, no. 2, pp. 1463-1474, 2020.

[24] N. Basol, H. Aygun, and S. S Gul, "Beneficial effects of edaravone in experimental model of amitriptyline-induced cardiotoxicity in rats," Naunyn-Schmiedeberg', vol. 392, pp. 1447-1453, 2019. 